steatosis being correlated with bigger values of SWV. Positive statistical correlations have been established between AST and SWV in the group of obese chidren and after-chemotherapy.

Conclusions ARFI allows SWV quantification of the SWV in strong correlation with the fibrosis stage, the hepatic steatosis and the liver changes after chemotherapy.

"This paper is partially supported by the Sectoral Operational Programme Human Resources Development, financed from the European Social Fund and by the Romanian Government under the contract number POSDRU/89/1.5/S/60782".

\section{GASTROINTESTINAL TRANSIT PATTERNS IN CHILDHOOD CHRONIC CONSTIPATION}

doi:10.1136/archdischild-2012-302724.0678

1.2,3YI Yik, ${ }^{4} \mathrm{DJ}$ Cook, ${ }^{4} \mathrm{DM}$ Veysey, ${ }^{4} \mathrm{CF}$ Tudball, ${ }^{4} \mathrm{BS}$ King, ${ }^{4} \mathrm{KA}$ Morris, ${ }^{4} \mathrm{TM}$ Cain, ${ }^{3}$ BR Southwell, 2,3,5JM Hutson. 'Department of General Surgery, Faculty of Medicine, University of Malaya, Kuala Lumpur, Malaysia; '2Department of Paediatrics, University of Melbourne; ${ }^{3}$ Gut Motility and Research Laboratory, Murdoch Children's Research Institute; ${ }^{4}$ Department of Biomedical Imaging; ${ }^{5}$ Department of Urology, Royal Childrens Hospital Melbourne, Melbourne, VIC, Australia

Background and Aims Constipation may be a part of a generalized gastrointestinal (GI) tract disorder. Nuclear transit scintigraphy (NTS) provides transit through the stomach, small bowel, colon and anorectum. This study aimed to determine different colonic and rectal transit patterns in children with chronic constipation (CC) and their association with upper GI tract disorders.

Methods A retrospective analysis of NTS (1999-2011) performed in children with intractable CC. The 48-hour NTS protocol involved Gallium-67 citrate milk drink with images acquired at 0-2 hrs (gastric emptying study) and at 6, 24, 30 and 48 hrs (small bowel \& colonic transit studies). The geometric centre calculation was based on $\%$ of radioactivity in each region of interest (ROI). Six ROIs were employed ( $1=$ pre-colonic, $2=$ ascending colon, $3=$ transverse colon, $4=$ descending colon, $5=$ recto-sigmoid colon and $6=$ toilet).

Results A total of 955 NTS was performed (1999-2011; 288 repeat \& 667 new studies). In the 603 children (284 female, 2-23yrs, mean $8.5 \pm 4.1 \mathrm{yrs}$ ) included for this study, $19 \%$ had normal colonic transit (NT), $52 \%$ slow colonic transit (ST) and $29 \%$ rapid proximal colonic transit (RT, Table 1). Only $1 / 3$ of children had AR. About $20 \%$ of children had delayed gastric emptying \& delayed small bowel transit.

Conclusions There are 3 distinct colonic transit patterns in children with CC: normal, slow \& rapid. About $1 / 3$ of children with CC had AR at $48 \mathrm{hrs} \&$ was associated with NT, ST and RT. In addition, 21-24\% children with CC had upper GI tract transit disorders.

\section{INFLAMMATORY CHANGES AND CERAMIDE PROFILES IN RAT LIVER AFTER FETAL ASPHYCTIC PRECONDITIONING AND PERINATAL ASPHYXIA}

doi:10.1136/archdischild-2012-302724.0679
1,2E Vlassaks, ${ }^{2} \mathrm{M}$ Nikiforou, ${ }^{1,3} \mathrm{JSH}$ Vles, 1,2E Strackx, ${ }^{1} \mathrm{P}$ Martinez-Martinez, ${ }^{2} \mathrm{BW}$ Kramer, ${ }^{1,2}$ AWD Gavilanes. 'Mental Health and Neuroscience, Maastricht University; ${ }^{2}$ Pediatrics, ${ }^{3}$ Child Neurology, Maastricht University Medical Center, Maastricht, The Netherlands

Background and Aims Fetal (FA) and perinatal asphyxia (PA) are major causes of neonatal morbidity and death worldwide. Although most studies are focused on the brain, FA and PA are known to be associated with multi-organ disease. Therefore, as part of the systemic impact, we aimed to investigate the hepatic inflammatory response after asphyxia.

Methods A clinical relevant rat model was used, inducing global asphyctic insults to reflect the human pathophysiology. At different time points (acute and chronic) after FA and PA, we assessed hepatic inflammation, ceramide signaling and hepatocellular damage. Additionally, we assessed whether the combination of both insults (preconditioning) would have any protective effect on the liver.

Results FA induced significant changes in inflammatory cytokines and ceramide metabolism genes with increased interleukin (IL)- $1 \mathrm{~b}$ mRNA at $6 \mathrm{~h}$, increased mRNA levels of IL-6, LAG1 homolog ceramide synthase 1 and ceramide transporters at $24 \mathrm{~h}$ and finally, increased acid sphingomyelinase and sphingomyelin synthase 1 mRNA at 96h. Also PA induced an inflammatory response, with increased IL- 6 and IL-10 levels $2 \mathrm{~h}$ after birth. The combination of FA and PA (preconditioning) attenuated the inflammatory response, reflecting comparable IL-6 and IL-10 levels as control animals. 8 months after birth, no significant differences between groups were observed in hepatic mRNA levels for all cytokines and ceramide enzymes. Nevertheless, markers for hepatocellular damage, AST and ALP, showed increased levels when animals experienced FA and PA.

Conclusions FA and PA induce acute changes in hepatic cytokine and ceramide levels which may lead to hepatocellular damage in later life.

\section{INTRA-ABDOMINAL TUMORS IN CHILDREN}

doi:10.1136/archdischild-2012-302724.0680

E Blevrakis, T Tavladaki, AM Spanaki, E Vasilaki, S llia, E Geromarkaki, MD Fitrolaki, G Briassoulis. PICU, University Hospital of Heraklion, Heraklion, Greece

Aim The objective of the present study was to observe the histopathological pattern of intra-abdominal tumors in children less than 15 years.

Material and Methods The study was carried out at the Department of Pediatric Intensive Care Unit, Heraklion, during a period of 7 years, from March 2005 to March 2012. The histopathological and demographic data of 15 intra-abdominal tumors of both sexes ( 8 boys and 7 girls) under 16 years of age was collected and analyzes to determine the various morphological types of intra-abdominal tumors in relation to age and sex.

Results Neuroblastoma was the most common tumor constituting $46.7 \%$ of all cases, followed by Wilms'tumor (26.7\%), hepatoblastoma (13.2), teratoma and granulosa cell tumor (6.7\%) each. Majority of the patients $73.3 \%$ were under 5 years of age.

Abstract 678 Table 1

\begin{tabular}{llcccccc}
\hline Proximal colonic transit & \multicolumn{2}{c}{ Gastric emptying } & Small bowel transit & $\begin{array}{c}\text { Distal colonic } \\
\text { transit }\end{array}$ & Anorectal transit \\
\hline & Normal & \multicolumn{1}{c}{ Delayed } & Normal & Delayed & Slow & Normal & retention (AR) \\
\hline Normal (NT) & $91(15 \%)$ & $23(4 \%)$ & $82(14 \%)$ & $32(5 \%)$ & - & $62(10 \%)$ & $52(9 \%)$ \\
Slow (ST) & $251(42 \%)$ & $63(10 \%)$ & $221(36 \%)$ & $93(16 \%)$ & - & $237(39 \%)$ & $77(13 \%)$ \\
Rapid (RT) & $135(22 \%)$ & $40(7 \%)$ & $156(26 \%)$ & $19(3 \%)$ & $21(4 \%)$ & $74(12 \%)$ & $80(13 \%)$ \\
TOTAL & $477(79 \%)$ & $126(21 \%)$ & $459(76 \%)$ & $144(24 \%)$ & $21(4 \%)$ & $373(61 \%)$ & $209(35 \%)$ \\
\hline
\end{tabular}

